\title{
Sequential deposition of microdroplets on patterned surfaces
}

DOI:

10.1039/C8SM01373J

\section{Document Version}

Accepted author manuscript

Link to publication record in Manchester Research Explorer

\section{Citation for published version (APA):}

Kant, P., Hazel, A., Dowling, M., Thompson, A. B., \& Juel, A. (2018). Sequential deposition of microdroplets on patterned surfaces. Soft Matter, 14(43), 8709-8716 . https://doi.org/10.1039/C8SM01373J

\section{Published in:}

Soft Matter

\section{Citing this paper}

Please note that where the full-text provided on Manchester Research Explorer is the Author Accepted Manuscript or Proof version this may differ from the final Published version. If citing, it is advised that you check and use the publisher's definitive version.

\section{General rights}

Copyright and moral rights for the publications made accessible in the Research Explorer are retained by the authors and/or other copyright owners and it is a condition of accessing publications that users recognise and abide by the legal requirements associated with these rights.

\section{Takedown policy}

If you believe that this document breaches copyright please refer to the University of Manchester's Takedown Procedures [http://man.ac.uk/04Y6Bo] or contact uml.scholarlycommunications@manchester.ac.uk providing relevant details, so we can investigate your claim.

\section{OPEN ACCESS}




\title{
Journal Name
}

\section{ARTICLE TYPE}

Cite this: DOI: $10.1039 / x x x x x x x x x x$

Received Date

Accepted Date

DOI: $10.1039 / x x x x x x x x x x$

www.rsc.org/journalname

\section{Sequential deposition of microdroplets on patterned surfaces ${ }^{\dagger}$}

\author{
Pallav Kant, ${ }^{a}$ Andrew Hazel, ${ }^{a}$ Mark Dowling ${ }^{b}$, Alice Thompson, ${ }^{a}$ and Anne Juel,,${ }^{c *}$
}

\begin{abstract}
We use a combination of experiments and numerical modelling to investigate the influence of physico-chemical-patterned substrates on the spreading of fluid deposited as a partially overlapping sequence of droplets via inkjet printing. Our investigation is motivated by the manufacture of polymeric organic light-emitting-diode displays, where the substrate is textured with a regular array of shallow recessed regions (pixels) that are highly wetting compared to the remainder of the substrate. We examine the roles of topography and wettability patterning separately and in combination. On a substrate with uniform wettability, we find that the presence of bounding side walls enhances the local spreading and facilitates fluid coverage of the entire recessed region, but containment within the pixel is not guaranteed. In contrast, wettability patterning alone leads to robust containment of the fluid within the wetting region, but fluid coverage is reduced in the absence of side walls. Our theoretical calculations use a simplified numerical model of fluid redistribution via purely capillary effects, augmented by a Cox-Voinov spreading law. The neglect of fluid viscosity in this model means that, after an initial period of agreement, the predicted evolution is faster than in the experiments. Nonetheless, the simplified model achieves excellent predictions both for the liquid morphologies and for the conditions required for successful pixel filling on substrates with topographical and wettability variations.
\end{abstract}

\section{Introduction}

Patterned surfaces with pre-defined micron-sized topographical features, wettability patterns or a combination of both, are commonly employed to manipulate small amounts of deposited liquid. Hence understanding the influence of these physicochemical patterns on fluid redistribution is essential in a variety of applications, e.g. tissue engineering ${ }^{\square, 2}$, design of microelectromechanical systems ${ }^{[3}$ and fabrication of micro-fluidic devices ${ }^{4}$. In this article we address the spreading on a patterned substrate of a small number of fluid droplets deposited such that new droplets partially overlap the pre-existing fluid mass; a situation that arises in the inkjet printing based manufacture of POLED (Polymeric Organic Light Emitting Diode) displays ${ }^{5-\underline{7}}$.

The fabrication of a POLED display via inkjet printing involves the deposition of successive layers of electroluminescent polymeric inks onto the display backplane ${ }^{8}$. Each layer is formed

\footnotetext{
a School of Mathematics, University of Manchester, Oxford Road, Manchester M13 9PL, United Kingdom.

${ }^{b}$ Cambridge Display Technology Limited, Unit 12, Cardinal Business Park, Godmanchester, Cambridgeshire PE29 2XG, United Kingdom (Company Number 02672530)

${ }^{c}$ School of Physics and Astronomy, University of Manchester, Oxford Road, Manchester M13 9PL, United Kingdom. E-mail: anne.juel@manchester.ac.uk

$\dagger$ Electronic Supplementary Information (ESI) available: [details of any supplementary information available should be included here]. See DOI: 10.1039/b000000x/
}

by linear sequential deposition of partially overlapping droplets that coalesce upon impact into liquid films (see Fig. 四) contained within specified regions of the substrate referred to as pixels. A pixel forms the smallest light emitting region on a display backplane and has a distinct shape depending on the design requirements, with overview and cross section shown in Fig. $\mathbf{\Xi}(\mathrm{a}, \mathrm{b})$. The main challenge associated with the inkjet process is to deposit a precise volume of liquid, typically a few tens of pico-litres, to achieve uniform and continuous thin films of the desired pixel shape which in turn solidify upon evaporation of the liquid content ${ }^{[\mathrm{IO}-\mathrm{II}}$. Since any variations in the ink volume or irregularities in the printed pattern shape lower the resolution as well as the performance of the device, fabrication of high resolution displays requires the ability to accurately control the spreading of the deposited fluid on the substrate. This is typically achieved by modifying the surface characteristics of a substrate via fabrication of geometrical ${ }^{[3-\sqrt{35}}$ or chemical ${ }^{5,6}$ patterns, which aid in controlling the spread of the deposited liquid. Previous studies about the influence of surface modifications have been predominantly focused on the formation of very long lines of ink, using linear grooves ${ }^{[16,[7}$ or pre-patterned wetting regions ${ }^{[1, \sqrt{18,} \text {, and moreover }}$ the reported observations are usually limited to the identification of the final equilibrium morphologies.

In this study, we use high-speed imaging techniques to investi- 


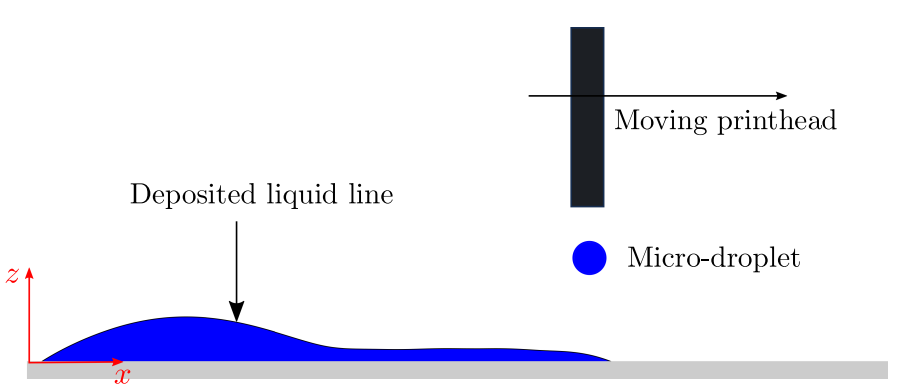

Fig. 1 Schematic side-view diagram of the experimental setup. Droplets of ink are deposited onto a substrate at a fixed frequency by a printhead moving at constant speed. The substrate may be topographically and chemically pre-patterned as indicated in Fig. [.

gate the time evolution of liquid morphologies formed from the sequential deposition of multiple droplets on patterned surfaces. Further, we combine experiments and numerical simulations of a simplified model of the fluid dynamics to investigate the separate roles of micron-sized topographical features and wettability patterns in the precise control of the spreading of fluid in this system. We find that compared to the existing complex 3-D numerical models ${ }^{[20, Z 1]}$, the simplified model presented here can be used as a computationally inexpensive predictive tool for similar classes of problems.

\section{Experimental methods}

In the experiments, equally spaced partially overlapping droplets, each of volume $V=7.6 \pm 0.4 \mathrm{pL}$, were deposited onto a substrate using a horizontally translating inkjet printhead as shown schematically in Fig. W. An industrial grade piezoelectric (dropon-demand) ink-jet print-head (SX3, Fujifilm Dimatix) was used to produce micro-droplets at a constant frequency $f=80 \mathrm{~Hz}$. Drops produced from the printhead had an in-flight radius $R_{f}=$ $\sqrt[3]{3 V / 4 \pi}=12.2 \mu \mathrm{m}$. The printhead was mounted on a linear motion stage (ANT95L, Aerotech) that allowed translation in the horizontal direction at varying speeds $\left(v=2.5-50 \mathrm{~mm} \mathrm{~s}^{-1}\right)$. The inter-drop distance $(\Delta x)$ between neighbouring droplets was controlled by varying the translation speed ( $v$ ) of the linear stage such that $\Delta x=v / f$. The deposited fluid was a Cambridge Display Technology (CDT) proprietary solution used in POLED printing with dynamic viscosity $\eta=6.25 \times 10^{-3}$ Pa s, density $\rho=1.066 \times 10^{3}$ $\mathrm{kg} \mathrm{m}^{-3}$ and surface tension $\sigma=44 \times 10^{-3} \mathrm{~N} \mathrm{~m}^{-1}$. The Weber number associated with the impact of the micro-droplets generated from the printhead was low, $W e=2 \rho V_{i}^{2} R_{f} / \sigma=2.4$, where $V_{i}=2.2 \mathrm{~m} \mathrm{~s}^{-1}$ is the vertical velocity of the droplet ${ }^{[2]}$, and accordingly no splashing was observed at the time of impact ${ }^{233}$. Bottom views of the liquid spreading on the substrate were recorded by a high speed camera (Photron SA3, $500 \mathrm{fps}$ ) kept in a fixed position, through a long distance zoom lens assembly (Navitar, with 10X objective, Mitutoyo) with a $90^{\circ}$ bend. The region of interest was illuminated from the top by a cold Xenon lamp light (Xenon NOVA 300, Karl Storz). All the experiments were performed under ambient laboratory conditions; the average evaporation rate per unit surface area of a sessile droplet of volume $7.6 \mathrm{pL}$ and contact angle $\sim 50^{\circ}$ was measured to be less than $0.168 \mu \mathrm{m} / \mathrm{min}$ $(<5 \%$ of the total volume per minute). (a)

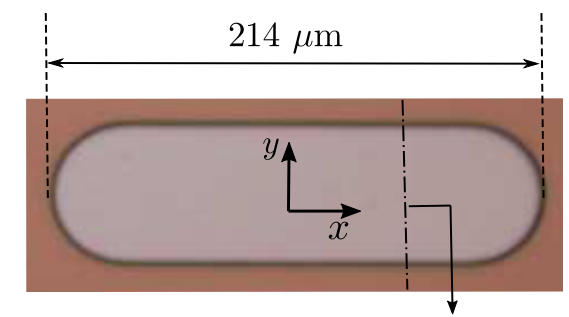

(b)

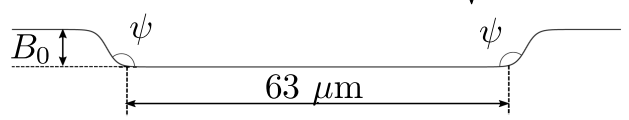

(c)

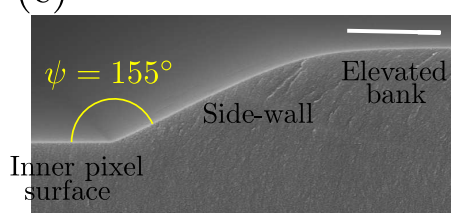

Type-I pixel side-wall

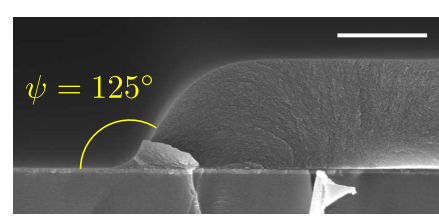

Type-II pixel side-wall (d)

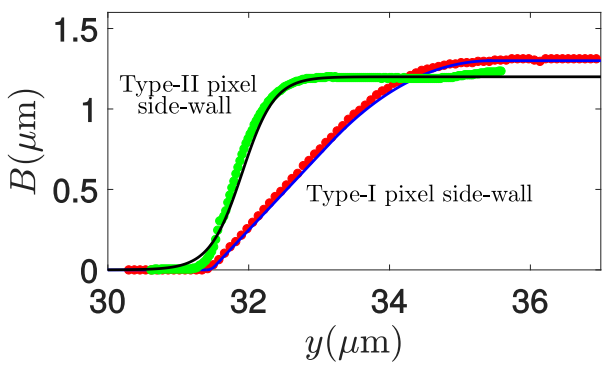

(e)

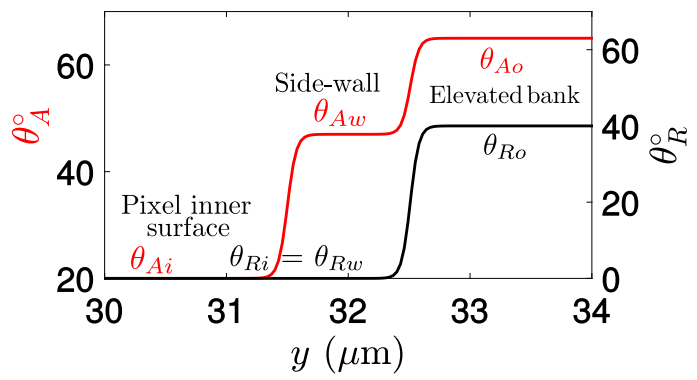

Fig. 2 (a) Top view of the stadium-shaped pixel used with both type-I and type-II substrates. (b) Schematic diagram of the profile of the pixel along the dot-dashed line in (a). The side wall of the recessed pixel is inclined with a corner angle $\psi=155^{\circ}$ (type-I) and $\psi=125^{\circ}$ (type-II), which were measured using scanning electron microscopy (SEM). The depth of the recessed pixel is $B_{0}=1.3 \mu \mathrm{m}$ (type-I) and $B_{0}=1.2 \mu \mathrm{m}$ (type-II). (c) SEM images of the side wall profiles of type-I and type-II. The horizontal scale bars each correspond to $1 \mu \mathrm{m}$. (d) Experimentally measured height profile (symbols) of a type-I (red) and II (green) side wall (obtained from the SEM images in (c)), and the fits to these measurements used in the numerical calculations (solid lines). (e) Contact angle profile applied in the model of a type-II pixel. Red line: advancing contact angles inside the pixel $\theta_{A i}$, on the elevated bank $\theta_{A o}$ and on the side wall $\theta_{A w}$. Black line: receding contact angles, $\theta_{R i}, \theta_{R o}$ and $\theta_{R w}$. 
Experiments were performed on two types of patterned substrates which were prepared in the clean room facility of CDT, type-I: surfaces with topographical features and uniform wettability, and type-II: surfaces with both topography and wettability patterns. Details of the fabrication process are provided in the ESI. The topographical features consisted of shallow stadiumshaped wells (pixels) bounded by sloping side walls of height $10 \%$ of the in-flight droplet radius; see Fig. $\mathbb{Z}(\mathrm{a}, \mathrm{b})$. For the purposes of our simulations, the geometry of the stadium-shaped pixels of types-I and II is modelled by approximating the side-walls by a smooth hyperbolic tangent profile, which closely matches the side-wall profile measured using SEM imaging; see Fig. Z (c,d).

The wettability of a type-I substrate is characterised by an advancing contact angle $1^{\circ} \leq \theta_{A} \leq 23^{\circ}$ and a receding contact angle $\theta_{R}<1^{\circ}$, both measured on its flat region. On type-II substrates the wettability variation is characterised by advancing and receding contact angles measured on the elevated surfaces (banks) outside the pixel boundary $\left(\theta_{A o}=65 \pm 2^{\circ}, \theta_{R o}=40 \pm 2^{\circ}\right)$, within the pixel boundary $\left(4^{\circ} \leq \theta_{A i} \leq 40^{\circ}, \theta_{R i}=0^{\circ}\right)$ and on the sloping sidewalls $\left(\theta_{A w}=47 \pm 2^{\circ}, \theta_{R w}=0^{\circ}\right)$. The direct measurement of the wetting properties of the sloped side-walls was not experimentally feasible due to the narrow width of the side wall $(\leq 1 \mu \mathrm{m})$. Therefore, separate substrates were fabricated using the proprietary photo resist that formed the side wall (see ESI), and both advancing and receding contact angles were measured on flat regions of these substrates. In our simulations, we approximate the wettability variation using combinations of different hyperbolic tangent profiles for both advancing and receding contact angles as shown in Fig. $\gtrsim(e)$.

\section{Mathematical model}

We performed numerical simulations for both types of substrates, and also used our theoretical model to investigate the effects of wettability patterning in the absence of topography (a configuration which we could not realise experimentally). We use a simplified modelling methodology which has been shown to successfully predict the evolution and final morphology of overlapping droplets printed on unpatterned substrates ${ }^{2.2}$ and for a single droplet printed inside a pixel with uniform wettability ${ }^{\text {24. }}$.

Following Thompson et al. ${ }^{\sqrt{22}}$ and Kant et $\mathrm{al}^{\sqrt{24}}$, we approximate the liquid morphology as a thin-film that evolves (quasi-statically) under only capillary forces driven by a dynamic spreading law that relates the speed of the contact line $U$ and the contact angle $\theta$. We introduce Cartesian coordinates, where the $x$ and $y$ axes are aligned with the length and width of the pixel, respectively, such that the incompressible fluid lies between the substrate at $z=B(x, y)$ and the free surface at $z=h(x, y, t)$. We define the footprint $\Omega(t)$ as the projection onto the $x y$ plane of the region where fluid and substrate meet at $z=B$. We consider the strong surface tension limit so that the fluid rearranges itself instantaneously in response to changes in the position of the contact line and the excess internal pressure $P(t)$ is spatially constant. Provided that at an instant in time, the shape of the footprint $\Omega(x, y, t)$ and the total volume $V$ of the liquid morphology are known, a unique solution $(h(x, y, t), P(t))$ is obtained by solving the linear equations

$$
P=-\sigma \cdot \nabla^{2} h, \quad V=\int_{\Omega}(h-B) \mathrm{d} x \mathrm{~d} y
$$

subject to the condition $h(x, y)=B(x, y)$ at the contact line, where $\partial \Omega$ denotes the boundary of the footprint. The side walls of the stadium-shaped pixel are approximated by a smooth 'tanh' profile; see Fig. $\boldsymbol{Q}(\mathrm{d}, \mathrm{e})$. The contact angle on $\partial \Omega(x, y, t)$ is given by $\theta=$ $-\vec{n} \cdot \nabla(h-B)$, where $\vec{n}$ is the two dimensional unit vector directed out of $\Omega(x, y, t)$.

A kinematic boundary condition, $\vec{n} \cdot \frac{\mathrm{d} R}{\mathrm{~d} t}=U(\theta)$, enables the advancing or receding motion of the contact line, with a normal velocity prescribed by the spreading law $U(\theta)$. At each time step, we calculate the contact angle, update the footprint with the kinematic condition, and then recalculate $h(x, y, t)$. The CoxVoinov spreading law ${ }^{25,26}$ appropriately describes the motion of the contact-line in our system ${ }^{24}$, and we parametrise it with experimental measurements of the advancing and receding contact angles and velocity scales $\left(U_{A}, U_{R}\right)$ measured inside the pixel and on the banks (see Table $\mathrm{U}$ ), so that

$$
U(\theta)=\left\{\begin{array}{lll}
U_{A}\left(\theta^{3}-\theta_{A}^{3}(x, y)\right), & \text { if } \quad \theta>\theta_{A}(x, y), \\
0, & \text { if } \quad \theta_{R}(x, y)<\theta<\theta_{A}(x, y), \\
U_{R}\left(\theta_{R}^{3}(x, y)-\theta^{3}\right), & \text { if } \theta<\theta_{R}(x, y) .
\end{array}\right.
$$

The equations are solved using a finite element method with piecewise quadratic approximation in space and a second-order implicit timestepping scheme implemented within the software library oomph-lib 27 . The domain is discretised via a triangular mesh that deforms as the fluid spreads, and so the mesh boundary remains fitted to the contact line. We can therefore obtain results for arbitrary contact-line shapes.

Upon deposition of a new droplet, the liquid footprint is updated to be the union of the regions wetted by the existing liquid and the newly deposited, partially overlapping droplet; and the total volume is increased accordingly - see Fig. B for a schematic representation of the droplet deposition scheme. The fluid pressure and height profile consistent with the updated footprint and fluid volume are then adjusted to ensure that the fluid is in static equilibrium.

We do not simulate the complex drop-scale interactions associated with the coalescence of each droplet with the already-printed fluid. Instead, based on experimental observations, we approximate the footprint of each new printed drop as a disc of radius $R_{p}=\phi R_{0}$ that partially overlaps the printed line, where $R_{0}$ is the radius of a drop just after the impact on a dry surface and $\phi$ is a parameter that approximates the effects of the coalescence with already-printed fluid; see Fig. B]. To measure $R_{0}$, we fit a circle to the perimeter of the liquid footprint visible in the first bottom-view image (recorded at $500 \mathrm{fps}$ ) immediately after the impact of the first droplet of a deposition sequence, estimated to be at $t=0.002 \mathrm{~s}$. For type I substrates with $\theta_{A}=7^{\circ}$, we obtain $R_{0}=25.97 \mu \mathrm{m}$ while for $\theta_{A}=23^{\circ}, R_{0}=23.48 \mu \mathrm{m}$. For the interior of type II substrates, $\theta_{A}=20^{\circ}$ and we measure $R_{0}=24.56 \mu \mathrm{m}$. These values of $R_{0}$ correspond to initial contact angles of between $30^{\circ}$ and $39^{\circ}$. For subsequent drops the value of $R_{p}$ and hence $\phi$ is measured from each image immediately after deposition; we 
Table 1 Values of $U_{A}$ and $U_{R}$ used in the numerical computations.

\begin{tabular}{cccc}
\hline \hline Substrate type & Wettability & $U_{A}\left(\mathrm{~ms}^{-1} \mathrm{rad}^{-3}\right)$ & $U_{R}\left(\mathrm{~ms}^{-1} \mathrm{rad}^{-3}\right)$ \\
\hline Type-I & $\theta_{A}=7^{\circ}, \theta_{R}=0^{\circ}$ & $2.266 \times 10^{-3}$ \\
Type-I & $\theta_{A}=23^{\circ}, \theta_{R}=0^{\circ}$ & $1.366 \times 10^{-3}$ & 0 \\
Type-II & $\theta_{A i}=20^{\circ}, \theta_{R i}=0^{\circ} ; \theta_{A o}=65^{\circ}, \theta_{R o}=40^{\circ}$ & $1.819 \times 10^{-3}$ & $7.276 \times 10^{-3}$ \\
\hline
\end{tabular}

(a)
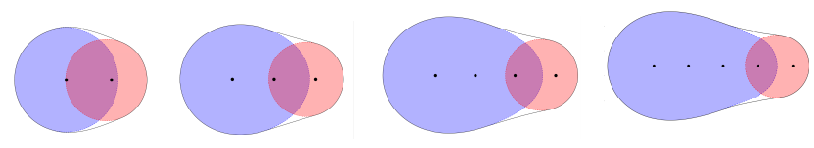

Smooth curve joining new footprint

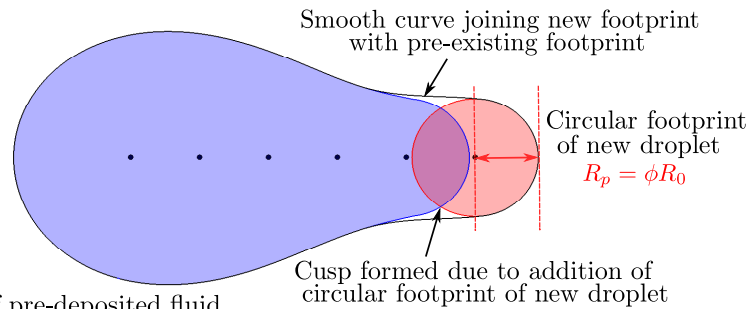

Footprint of pre-deposited fluid

(b)

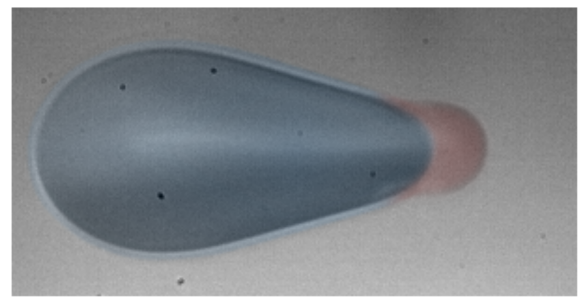

Fig. 3 (a) Schematic top view of droplet deposition. The blue shaded region represents the footprint of the fluid layer already on the substrate and the circular red region of radius $R_{p}=\phi R_{0}$ corresponds to the footprint of the newly deposited droplet. Black dots indicate the centres of mass of the deposited droplets, which are separated by $\Delta x$. (b) Experimentally observed footprint of the liquid just after the deposition of a new droplet. The red shaded region is the footprint of the deposited droplet and the blue shaded region corresponds to the footprint of the fluid already on the substrate. In the model, smoothing was applied to the contact line in the cusped region where the contact lines of both fluid regions intersect, see (a), in order to mimic the smooth contact line observed experimentally in (b); see section III of the ESI for more details. find that $\phi$ remains approximately constant across all the contact angles studied with an average value of $\phi=0.83 \pm 0.08$. In the simulations, we use $R_{0}$ for each sequence based on the measured values for that substrate, with $\phi=1$ for the first drop in each sequence and $\phi=0.83$ for all subsequent drops.

\section{Results}

\subsection{Influence of topographical patterns}

In Fig. 田, we compare the experimentally observed evolution of the liquid mass formed from the sequential deposition of five droplets with $\Delta x=25 \mu \mathrm{m}$ along the long centreline of a type-I recessed pixel $\left(\theta_{A}=7^{\circ}\right.$ and $\left.\theta_{R}=0^{\circ}\right)$ and on a flat region away from the pixels on exactly the same substrate, which ensures that the wetting properties are near identical. The initial development is very similar on both surfaces. The first two droplets of the sequence merge to form a large sessile droplet with an approximately circular footprint and a reduced curvature compared with a single droplet. The larger curvature of each incoming droplet results in excess capillary pressure within the newly coalesced droplet, which drives liquid back into the pre-existing fluid layer to form a bulge at the upstream end of the deposition sequence; see Fig. S3 (ESI) for a side-view sequence of this process. On this substrate, the contact line cannot recede because $\theta_{R} \approx 0^{\circ}$ and therefore, on the flat surface, the deposited liquid evolves into a thin film with a non-circular footprint which retains the length of the initial printed region (Fig. 目(a))

In contrast, inside the pixel, the liquid evolves into a thin film that covers the entire pixel surface, owing to the interaction between the liquid and the sloping side-walls (Fig. G(b)). Locally, the sloping side walls lead to an increased effective contact angle which, via the spreading law, leads to faster spreading. This means that the fluid on the sidewalls advances faster than that in the centre of the pixel, promoting the growth of two side-wall wetting regions, in which the fluid preferentially spreads along the side walls. Once all droplets have been deposited so that the liquid volume remains constant, rivulets form at the extremities of the side-wall wetting regions and grow along the boundary to cover its perimeter. Kant et al ${ }^{\sqrt{24}}$ showed that the development of related rivulets in single-drop spreading within a pixel is closely related to the phenomenon of unbounded capillary rise in a tube of polygonal cross-section in the absence of gravity ${ }^{28}$. Similar enhanced spreading of liquid along the corners on a microstructured surfaces has been reported by also reported by Seemann et al ${ }^{[33}$ and Khare et al $\stackrel{[16}{ }$. We observed the formation of rivulets along the pixel side-walls on substrates with $\theta_{A}<1^{\circ}$ and $\theta_{A}=7^{\circ}, 11^{\circ}$ but not for $\theta_{A}=23^{\circ}$, see Fig. 5(b), which is consistent with the capillary rise threshold of $12.5^{\circ}$ predicted for the slope angle of the side-walls here. The spreading of liquid along the boundaries prevents the formation of a bulging morphology as 
(a)

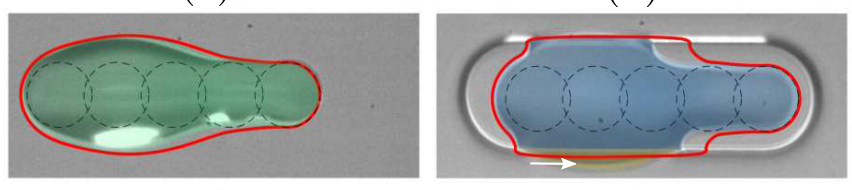

(a)
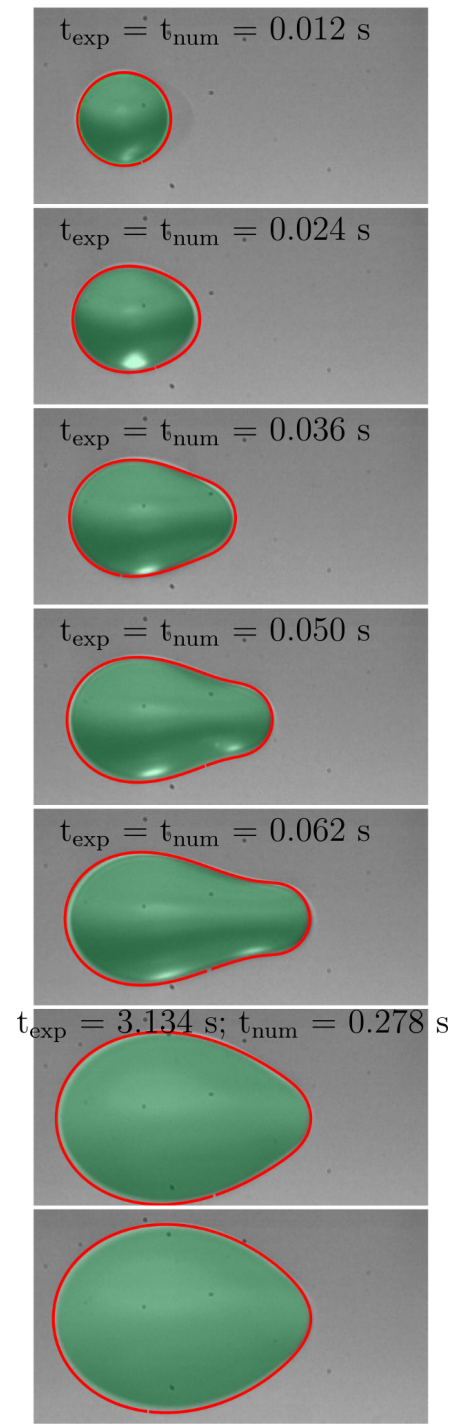

$\mathrm{t}_{\text {exp }}=25.184 \mathrm{~s} ; \mathrm{t}_{\mathrm{num}}=0.624 \mathrm{~s}$

Fig. 4 Evolution of liquid footprints formed during the deposition of five droplets $(\Delta x=25 \mu \mathrm{m})$ on a type-I substrate with $\theta_{A}\left(\theta_{R}\right)=7^{\circ}\left(0^{\circ}\right)$. In (a) droplets are deposited on one of the flat regions outside the pixels; in (b) droplets are deposited along the horizontal centreline of a recessed pixel. The first five snapshots in each sequence are spaced at a constant time interval equivalent to the printing frequency. In the first four of these, a new drop is about to be printed. The final shape in each sequence is an equilibrium state. Fluid spillage outside the boundary of the pixel in the final three images in (b) is highlighted in yellow and also indicated by the white arrows. Numerical predictions for the footprint shape are shown as red contours; note that for (b) the computations are terminated when the rivulets growing around the curved walls of the pixel first intersect.

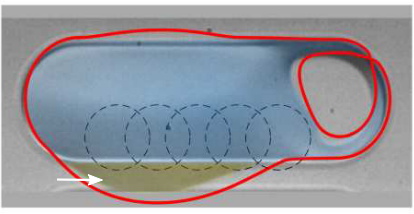

(c)

Fig. 5 Final states of the liquid footprints formed from the deposition of five droplets at $\Delta x=35 \mu \mathrm{m}$ on a type-I substrate with $(\mathrm{a}, \mathrm{b}) \theta_{A}=23^{\circ}$ (c) $\theta_{A}=7^{\circ}$, and in each case $\theta_{R}=0^{\circ}$. Drops are placed (a) on a flat region of the substrate outside a pixel, (b) along the horizontal centreline of a pixel and (c) just inside the pixel boundary. The dashed black circles indicate the deposition location of the droplets. The regions of fluid spillage outside the pixel boundaries in $(b, c)$ have been coloured in yellow and also indicated by the white arrows. For $(a, b)$, the liquid footprint is shown at equilibrium, with the equivalent numerically computed equilibrium states shown in red. For (c), the experimental and numerical results are shown at the moment the contact line first self-intersects.

observed on the flat surface. It thereby promotes the containment of the liquid inside the pixel boundary, although minor spillage is observed in the last three frames of Fig. U(b).

Numerical computations performed with the experimental parameter values capture the main features of the liquid redistribution observed experimentally (red contours overlaid on the experimental images in Fig. (A). The model quantitatively predicts the initial development on type-I substrates in both uniform regions and inside the pixels. For $t_{\exp }>0.0625 \mathrm{~s}$, however, the numerical and experimental timescales diverge and the liquid spreads faster in the computations in both cases. The model does not include any viscous dissipation, which would be expected to slow and moderate the evolution both in the thinning bulk of the liquid layer and also in the rivulets that grow along the side-walls in type-I substrates ${ }^{24}$. On flat substrates, no rivulets form and the model accurately predicts the equilibrium state of the liquid, despite the difference in time scales. In contrast, when close to pixel walls on the type-I substrates, the neglect of viscous effects in the model leads to the development of thicker rivulets and enhanced spillage onto the elevated banks compared with the experiment.

For the less-wetting surfaces, $\theta_{A}=23^{\circ}$, with $\theta_{A}$ above the capillary rise threshold, the side walls lead to a localized deformation of the contact line, but not preferential wetting of the the side walls. Consequently, the overall equilibrium state formed inside the pixel (Fig. 5b) was similar to that formed on a flat surface (Fig. 5la), except for the wetting regions along the side walls that promoted partial containment of the fluid. However, not all the fluid printed within the pixel could be contained inside its boundary and this resulted in minor spillage onto the elevated banks.

These results demonstrate that for the pixel configuration in Fig. ㄱ, topographical features alone are not sufficient to confine the spreading of the deposited liquid to the inside of the pixel 
region. Moreover, the containment of the deposited liquid relies sensitively on the accurate positioning of the droplets. For the size of pixel and droplet volume used, even a small offset in the deposition location of the droplets from the centreline of the pixel (as low as $\sim 10 \%$ of the width of the pixel) was sufficient for a droplet to be partially deposited outside the pixel boundary, see Fig. 5(c), where five droplets $(\Delta x=25 \mu \mathrm{m})$ have been deposited on the same substrate as in Fig. 田 $\left(\theta_{A}=7^{\circ}, \theta_{R}=0^{\circ}\right)$. The absence of receding contact line motion on this substrate meant that considerable spillage occurred outside the pixel boundary despite the side-wall rivulets aiding containment - a significant limitation in the manufacture of high-resolution display devices. Although this spillage could be mitigated by the use of smaller droplets, their reduced volume would in turn restrict the range of contact angles for which the droplets would spread sufficiently to cover the whole area of the pixel, which is a necessary requirement to achieve high performance displays. It is possible that using more complex topographical features to form sidewalls could prevent spillage. Li et al ${ }^{\sqrt{29}}$ were able to direct the spreading of $\sim 5 \mu L$ droplets using topographic variations of similar widths and lengths, but of greater depths $(\sim 5 \mu \mathrm{m})$ and geometric complexity than our pixels. At present it is not possible to manufacture these features at the scales required to have the same effect on the $\sim 7 p L$ droplets in our study. An alternative and robust means of eliminating this sensitivity to droplet positioning would be to enable the recession of the contact line outside the pixel boundary through significantly increased values of both $\theta_{A o}$ and $\theta_{R o}$.

\subsection{Influence of wettability patterning}

Having established the predictive power of the model, we shall use it to explore the role of wettability patterning in the absence of topographic variation because these types of surfaces were difficult to prepare using the fabrication method adopted in this study. In Fig. 6, we show the numerically computed evolution of a liquid footprint formed from the sequential deposition of seven droplets with $\Delta x=35 \mu \mathrm{m}$ along the centreline of a stadiumshaped pixel $\left(\theta_{A i}=20^{\circ}, \theta_{R i}=0^{\circ}\right)$, which is bounded by a lesswetting surface with high values of the advancing and receding contact angles $\left(\theta_{A o}=65^{\circ}, \theta_{R o}=40^{\circ}\right)$. The initial development of the liquid morphology within this type of pixel is qualitatively similar to that observed on a flat surface; see Fig. 4(a). Fluid redistribution results in a bulge at the upstream end that thins into a narrower rivulet following the deposition of the last four droplets. The less-wetting surface surrounding the pixel acts as a barrier to spreading, thus confining the liquid inside the pixel. In addition, the high receding contact angle outside the pixel boundary ensures that any droplets that are deposited partially outside the pixel boundary (first and last of the sequence) are pushed back inside it; see Fig. $\square$ for an experimental illustration of this process. This clearly demonstrates that wettability patterning provides an effective means of constraining the spreading of a deposited liquid to within a pixel region as long as the droplets are deposited at least partially within the pixel. However, the absence of side walls (and associated local increases of the contact angle) means that liquid spreading is not enhanced along the pixel boundaries.

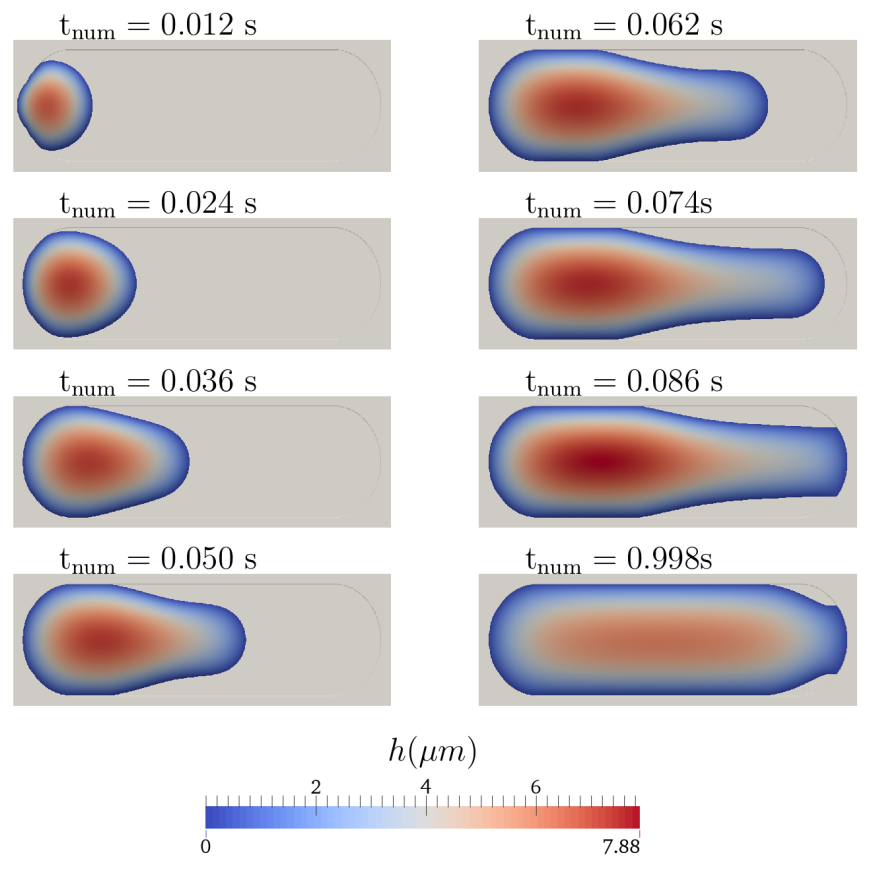

Fig. 6 Numerically computed drop-by-drop evolution of a liquid footprint formed during the deposition of seven partially overlapping droplets at $\Delta x=35 \mu \mathrm{m}$, inside a chemically-patterned flat pixel with $\theta_{A i}\left(\theta_{R i}\right)=20^{\circ}\left(0^{\circ}\right)$ and $\theta_{A o}\left(\theta_{R o}\right)=65^{\circ}\left(40^{\circ}\right)$. The time interval between snapshots is equal to the printing frequency.

Hence, at equilibrium the pixel remains under-filled (Fig. [, last image) in contrast with experiments and computations on type-II substrates with the same values of $\Delta x$ and contact angles, which we discuss below.

\subsection{Influence of both wettability and topography patterning} The sequence of experimental snapshots in Fig. 8 shows the dropby-drop evolution of the liquid footprint during the same deposition sequence as in Fig. 6 along the centreline of a type-II pixel; both sequences use the same non-uniform contact angle values inside and outside the pixel boundary, but in the latter there is additionally topographic variation. The key difference in the evolution of the liquid morphology in Fig. 8 compared to Fig. 6 is the wetting of the pixel boundary.

The locally enhanced values of the contact angle on the sloping side walls result in a locally distorted footprint shape in the vicinity of the side wall, which concurs with previously observed static wetting morphologies on microstructured surfaces $\underline{\text { [3, } 16}$. This wetting front is able to propagate along the side walls, resulting in the eventual liquid coverage of the entire pixel. This effect is illustrated in Fig. 9 where the numerical computation of a liquid line spreading inside a type-II pixel (Fig. Qb) shows that the local distortion of the footprint is associated with a local increase in the contact angle which enhances spreading of liquid via wetting fronts eventually leading to the liquid coverage of the entire typeII pixel (Fig. 9c). This effect is absent in the case where a liquid line spreads inside a pixel without sloping side-walls (Fig. Q2a,c).

Numerical predictions of the evolving fluid morphology are 

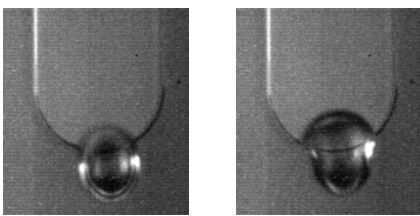

$\mathbf{t}=\mathbf{5} \times 10^{-4} \mathrm{~s}$ $\mathbf{t}=15 \times 10^{-4} \mathbf{s}$

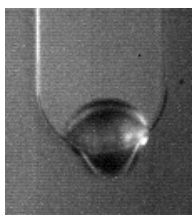

$\mathbf{t}=25 \times 10^{-4} \mathrm{~s}$

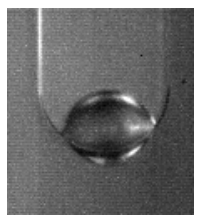

$\mathbf{t}=35 \times 10^{-4} \mathbf{s}$

Fig. 7 Sequence of images illustrating the receding motion of the contact line for a droplet deposited partially outside a type-II pixel with $\theta_{A i}\left(\theta_{R i}\right)=20^{\circ}\left(0^{\circ}\right)$ within the pixel boundary and $\theta_{A o}\left(\theta_{R o}\right)=65^{\circ}\left(40^{\circ}\right)$. The large value of the receding contact angle outside the pixel boundary means that any droplet that is partially deposited outside the pixel boundary is pushed back into it. These images were recorded in bottom view at $2000 \mathrm{fps}$.
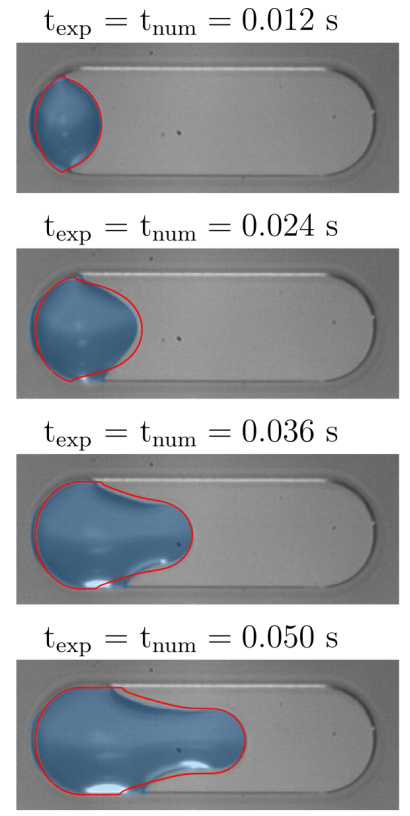
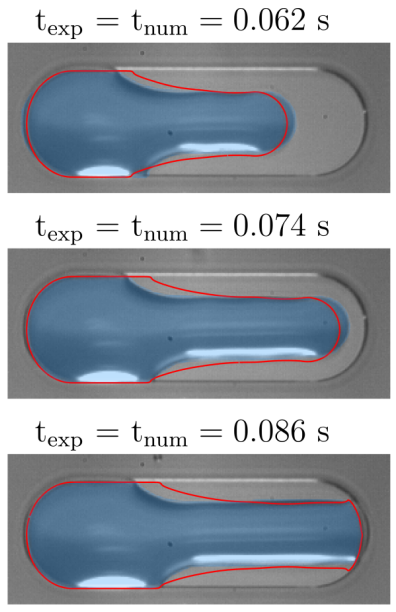

$\mathrm{t}_{\text {exp }}>120.000 \mathrm{~s} ; t_{\text {num }}=0.818 \mathrm{~s}$

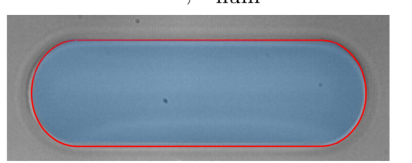

Fig. 8 Drop-by-drop evolution of the liquid footprint during the deposition of seven partially overlapping droplets at $\Delta x=35 \mu \mathrm{m}$, in a topographically and chemically patterned pixel with $\theta_{A i}\left(\theta_{R i}\right)=20^{\circ}\left(0^{\circ}\right)$ and $\theta_{A o}\left(\theta_{R o}\right)=65^{\circ}\left(40^{\circ}\right)$. The first seven images are equally spaced in time, with a new drop about to be deposited in the first six; the final image shows the equilibrium state. The blue shaded regions indicate the experimentally measured liquid footprint, while the red contours show the numerically-predicted footprint.

(a)

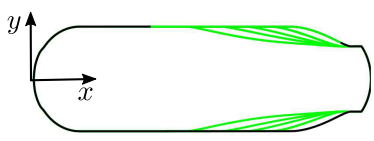

(b)

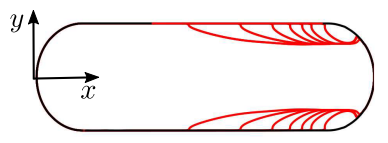

(c)

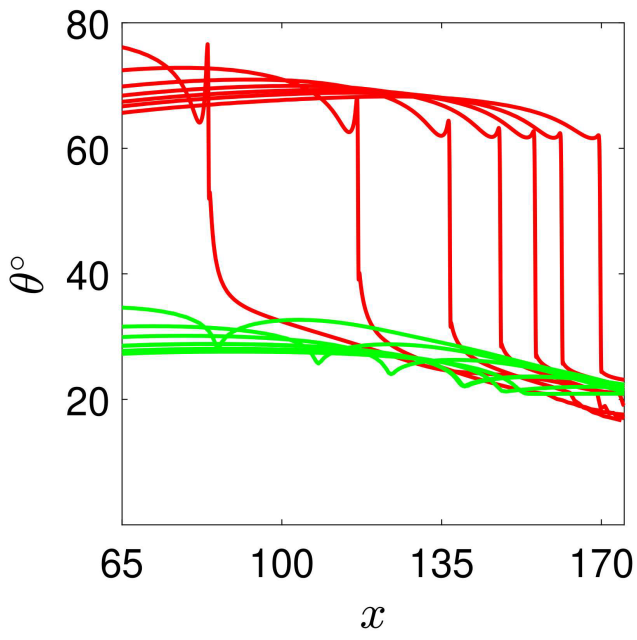

Fig. 9 (a,b) Comparison of the numerically computed late-time evolution of liquid lines formed from the deposition of seven droplets at $\Delta x=35 \mu \mathrm{m}$ inside the pixels shown in Fig. $\mathbf{6}$ (wettability pattern only) and Fig. $\mathbf{8}$ (type-Il pixel). The black outlines indicate the equilibrium states of the footprint in both cases. (c) Temporal evolution of the contact angle along the highlighted sections of the liquid footprints shown in $(a, b)$. The interaction between liquid and pixel side-walls in (b) results in a locally distorted footprint shape which is also associated with a sharp increase in the contact angle, approximately equal to the change in angle of the substrate, at the wetting front as shown in (c). 
shown in Fig. 8 with red contours overlaid on the experimental images. The overall evolution of the liquid footprint predicted by the numerical model is in excellent agreement with the experiments. The model correctly reproduces the formation of the bulge at the upstream end of the printed line and the eventual equilibrium state of the liquid line. Also, the overall motion of the wetting fronts formed along the side walls is correctly captured by the model, although there is a slight mismatch in the position of these wetting fronts compared to experiments. This suggests that our approximation of the side walls as uniformly wetting might not be sufficiently accurate; see Fig. ㄴ. The noticeable difference in the numerical and experimental timescales at late times can again be attributed to the absence of bulk viscous effects in the model ${ }^{24}$.

\section{Conclusion}

In summary, we have investigated the spreading of multiple droplets deposited sequentially in a straight line on surfaces with specific physico-chemical patterns, motivated by POLED display manufacture. We find that topographical patterning in the form of a shallow (depth $\sim 10 \%$ of in-flight droplet radius) recessed region with sloped side walls promotes spreading of liquid via the local increase in contact angle, which drives the formation of an advancing wetting front at the side wall. If the equilibrium contact angle inside the pixel is below a threshold value, this wetting front takes the form of thin rivulets that grow along the side walls through the mechanism of capillary rise. Irrespective of whether rivulets form, the enhanced spreading is not sufficient to ensure the containment of fluid within the pixel, which also depends sensitively on the placement of the droplets. In contrast, wettability patterning alone enables robust fluid containment but does not enhance spreading. Hence, microscopic topographical features and wettability patterning are both required to achieve robust containment of fluid without compromising pixel filling.

Although we have considered a limited set of pixel geometries, we expect the identified mechanisms to operate over a broader range of patterned substrates, and across a wide range of drop sizes and spacings, provided that the deposited fluid encounters the changes in topography and/or wettability. In particular, we expect that the combination of both wettability and topographical patterning will lead to more robust manipulation of the deposited liquid compared to either acting in isolation. Moreover, we have developed a simplified and computationally inexpensive numerical model that predicts the evolution of a sequence of partially overlapping droplet deposited on patterned surfaces, and may serve as a useful design tool for designing and modifying printing strategies in the POLED as well as LCD (Liquid Crystal Display) ${ }^{[30}$ manufacturing industries.

\section{Conflicts of interest}

There are no conflicts to declare.

\section{Acknowledgements}

The authors would like to thank Malcolm Walker and Carl Dixon for technical support while building the experimental facility. We acknowledge funding from the University of Manchester and
Cambridge Display Technology Ltd. We are also very grateful to Cambridge Display Technology Ltd. for providing the substrates required for this study.

\section{Notes and references}

1 G. M. Whitesides, E. Ostuni, S. Takayama, X. Jiang and D. E. Ingber, Annu. Rev. Biomed. Eng., 2001, 3, 335-373.

2 C. S. Chen, M. Mrksich, S. Huang, G. M. Whitesides and D. E. Ingber, Science, 1997, 276, 1425-1428.

3 A. del Campo and E. Arzt, Chem. Rev., 2008, 108, 911-945.

4 J. C. McDonald, D. C. Duffy, J. R. Anderson, D. T. Chiu, H. Wu, O. J. Schueller and G. M. Whitesides, Electrophoresis, 2000, 21, 27-40.

5 J. Wang, Z. Zheng, H. Li, W. Huck and H. Sirringhaus, Nat. Mater., 2004, 3, 171.

6 H. Sirringhaus, T. Kawase, R. Friend, T. Shimoda, M. Inbasekaran, W. Wu and E. Woo, Science, 2000, 290, 21232126.

7 M. Kuang, L. Wang and Y. Song, Adv. Mater., 2014, 26, 69506958.

8 J. Halls, Inf. Disp, 2005, 2, 10-15.

9 T. Shimoda, K. Morii, S. Seki and H. Kiguchi, MRS Bull., 2003, 28, 821-827.

10 P. C. Duineveld, J. Fluid Mech., 2003, 477, 175-200.

11 D. Soltman and V. Subramanian, Langmuir, 2008, 24, 2224 2231.

12 J. Stringer and B. Derby, Langmuir, 2010, 26, 10365-10372.

13 R. Seemann, M. Brinkmann, E. J. Kramer, F. F. Lange and R. Lipowsky, Proc. Natl. Acad. Sci. USA, 2005, 102, 18481852.

14 S. Herminghaus, M. Brinkmann and R. Seemann, Ann. Rev. Mater. Res, 2008, 38, 101-121.

15 C.-M. Keum, I.-H. Lee, H.-L. Park, C. Kim, B. Lüssem, J. S. Choi and S.-D. Lee, J. Appl. Phys., 2017, 121, 244902.

16 K. Khare, S. Herminghaus, J.-C. Baret, B. M. Law, M. Brinkmann and R. Seemann, Langmuir, 2007, 23, 1299713006.

17 C. E. Hendriks, P. J. Smith, J. Perelaer, A. M. Van den Berg and U. S. Schubert, Adv. Funct. Mater., 2008, 18, 1031-1038.

18 A. A. Darhuber, S. M. Troian, S. M. Miller and S. Wagner, J. Appl. Phys., 2000, 87, 7768-7775.

19 H. Gau, S. Herminghaus, P. Lenz and R. Lipowsky, Science, 1999, 283, 46-49.

20 W. Lee and G. Son, Computers \& Fluids, 2011, 42, 26-36.

21 A. Dalili, S. Chandra, J. Mostaghimi, H. C. Fan and J. C. Simmer, J. Colloid Interf. Sci., 2014, 418, 292-299.

22 A. B. Thompson, C. R. Tipton, A. Juel, A. L. Hazel and M. Dowling, J. Fluid Mech., 2014, 761, 261-281.

23 C. W. Visser, P. E. Frommhold, S. Wildeman, R. Mettin, D. Lohse and C. Sun, Soft Matter, 2015, 11, 1708-1722.

24 P. Kant, A. L. Hazel, M. Dowling, A. B. Thompson and A. Juel, Phys. Rev. Fluids, 2017, 2, 094002.

25 R. Cox, J. Fluid Mech., 1986, 168, 169-194.

26 O. Voinov, Fluid Dyn., 1976, 11, 714-721. 
27 M. Heil and A. L. Hazel, in Fluid-structure interaction, Springer, 2006, pp. 19-49.

28 P. Concus and R. Finn, Proc. Natl. Acad. Sci. USA, 1969, 63, 292-299.

29 J. Li, X. Zhou, J. Li, L. Che, J. Yao, G. McHale, M. K. Chaudhury and Z. Wang, Science Advances, 2017, 3, eaao3530.

30 C.-T. Chen, K.-H. Wu, C.-F. Lu and F. Shieh, J. Micromechanics Microengineering, 2010, 20, 055004. 